\title{
Hydrodynamic Fluctuations and Stokes' Law Friction
}

\author{
Robert Zwanzig
}

(September 3, 1964)

\begin{abstract}
The frictional force on a Brownian motion particle can be expressed by means of the timecorrelation of the fluctuating force on the particle. We show that this method, applied to a spherical particle in a viscous incompressible fluid, leads to Stokes' Law. The calculation is based on the theory of hydrodynamic fluctuations due to Landau and Lifshitz, and on a hydrodynamic theorem due to Faxen.
\end{abstract}

The subject of this article is the connection between two different methods for calculating the frictional force on a Brownian motion particle. One method is based on the statistical mechanical theory of irreversible processes, and involves evaluation of a certain time-correlation formula. The other method is based on macroscopic hydrodynamics, and requires solution of the Navier-Stokes equation.

For simplicity we restrict the discussion to the special case of a spherical particle of radius $a$ moving slowly through a viscous incompressible fluid. The viscosity coefficient is $\eta$.

In the hydrodynamic theory, the frictional force $\mathbf{F}$ on a sphere moving with constant velocity $\mathbf{v}$ is given by Stokes' law,

$$
\begin{aligned}
& \mathbf{F}=-\zeta \mathbf{v}, \\
& \zeta=6 \pi \eta a .
\end{aligned}
$$

In the molecular theory, eq (1) is unchanged; but the friction constant is now given by a time-correlation formula. This formula was obtained first by Kirkwood [1]. ${ }^{1} \quad$ A more precise and general treatment has been given by Lebowitz and Rubin [2].

The time-correlation formula for $\zeta$ is

$$
\zeta=\frac{1}{3 k_{B} \mathrm{~T}} \lim _{\epsilon \rightarrow 0} \int_{0}^{\infty} d t e^{-\epsilon t}\langle\mathbf{F}(0) \cdot \mathbf{F}(t)\rangle .
$$

In this expression, $\mathbf{F}(t)$ is the total force exerted on the sphere at time $t$ by the molecules in the surrounding fluid. The time dependence of $\mathbf{F}(t)$ is determined by solution of the molecular equations of motion, subject only to the condition that the spherical particle is held fixed in position. The angular bracket \langle\rangle denotes an average over a thermal equilibrium ensemble at temperature $T$. Boltzmann's constant is $k_{B}$.

${ }^{1}$ Figures in brackets indicate the literature references at the end of this paper.
The time-correlation formula (3) is known to be much more general than Stokes' formula (2). For example, Green [3] and Mazo [4] have shown that it gives the correct expression for the friction constant in a Rayleigh gas, where the molecular mean free path is much greater than the radius of the sphere. Stokes' formula, having been derived only in the hydrodynamic limit (mean free path much smaller than the radius of the sphere), is not valid for a Rayleigh gas.

In this article we show the equivalence of (2) and (3) under conditions where the hydrodynamic result is valid. Our discussion is based on the statistical theory of hydrodynamic fluctuations, as set forth by Landau and Lifshitz [5].

In the Landau-Lifshitz theory, as applied to a viscous incompressible fluid at low Reynolds number, the equations of motion are the conservation law

$$
\nabla \cdot \mathbf{v}=0
$$

and the generalized Navier-Stokes equation

$$
\rho \frac{\partial \mathbf{v}}{\partial t}=-\nabla p+\eta \nabla^{2} \mathbf{v}+\nabla \cdot \mathbf{S}
$$

with the convective inertial terms omitted because of the low Reynolds-number application. The pressure is $p$; the fluid density is $\rho$; and $\mathbf{S}$ is the fluctuation of the stress tensor away from the value it takes on when the fluid is in local thermodynamic equilibrium (i.e., the deviation from the usual Navier-Stokes stress tensor).

In the language of Brownian motion theory, eq (5) may be regarded as a Langevin equation. The divergence of $\mathbf{S}$ represents the fluctuating force acting on the fluid. This force is not specified exactly, but only in a statistical way. In particular, its mean value vanishes, and its second moment is given by

$$
\begin{array}{r}
\left\langle\mathbf{S}_{i k}\left(\mathbf{r}_{1}, t_{1}\right) \mathbf{S}_{l m}\left(\mathbf{r}_{2}, t_{2}\right)\right\rangle=2 k_{B} T \eta\left[\delta_{i l} \delta_{k m}+\delta_{i m} \delta_{k l}\right. \\
\left.-\frac{2}{3} \delta_{i k} \delta_{l m}\right] \times \delta\left(\mathbf{r}_{1}-\mathbf{r}_{2}\right) \times \delta\left(t_{1}-t_{2}\right) .
\end{array}
$$


(We have left out certain terms containing the coefficient of volume viscosity, since we are concerned with only incompressible fluids.)

Note that the correlation is taken to be local in space and time, as indicated by the delta functions in eq (6). This is an approximation which must fail for molecular times and distances; but because we are interested in only macroscopic times and distances, the approximation is good enough.

Because eq (5) is a Langevin equation, the probability distribution of velocities is given by a certain Fokker-Planck equation; this connection is quite familiar in the theory of Brownian motion. The appropriate Fokker-Planck equation is in fact the basic kinetic equation of Green's theory of irreversible processes in fluids [6].

As in the usual hydrodynamic derivation of Stokes' law, we assume all macroscopic processes to be so slow that the time derivative in eq (5) can be neglected.

Fluctuations in the local stress tensor give rise to fluctuations in the local pressure and velocity fields, and consequently to fluctuations in the total force on a sphere. When the fluid is at equilibrium, the mean velocity vanishes and the mean pressure is spatially uniform; so the mean force on the sphere vanishes. Thus we need to take account of only deviations from the mean behavior of the fluid.

To find the actual velocity and pressure fields associated with the fluctuating stress tensor, it appears at first that we must solve the hydrodynamic eqs (4) and (5) subject to the boundary condition $\mathbf{v}(\mathbf{r}, t)=0$ on the surface of the sphere. This calculation is a moderately difficult one. Fortunately, however, one can find the total force on the sphere using only the "unperturbed" velocity field that prevails in the absence of the sphere, by means of a remarkable theorem due to Faxen [7]. According to this theorem, the force $\mathbf{F}(t)$ on a sphere fixed at the origin, caused by an unperturbed velocity field $\mathbf{v}(\mathbf{r}, t)$, is

$$
\mathbf{F}(t)=6 \pi \eta a \cdot \frac{1}{4 \pi} \oint d \Omega \mathbf{v}(\mathbf{r}, t) .
$$

This equation evidently resembles Stokes' law, except that the velocity of the sphere has been replaced by the negative of the unperturbed velocity of the fluid, averaged over the surface of the sphere. $\quad(\oint d \Omega / 4 \pi$ denotes an average over all angles.)

It is easy to see that Stokes' law is a special case of Faxen's theorem.

The only conditions needed for the validity of Faxen's theorem are the ones we have already imposed: incompressibility, and omission of the time derivative in the Navier-Stokes equation.

To apply Faxen's theorem, we need the fluctuating velocity field for a medium that is at equilibrium, spatially uniform (no spherical particle present!), and infinite in extent. The calculation of this velocity field is performed easily by means of Fourier transforms.

Thus, we write any function $f(\mathbf{r})$ of position $\mathbf{r}$ as an integral,

$$
f(\mathbf{r})=\int d^{3} k f_{k} \exp i \mathbf{k} \cdot \mathbf{r} .
$$

In particular, the Fourier transform of eq (6) is

$$
\begin{aligned}
& \left\langle S_{i k}\left(\mathbf{k}_{1}, t_{1}\right) S_{l m}\left(\mathbf{k}_{2}, t_{2}\right)\right\rangle \\
& =\frac{2 k_{B} T \eta}{(2 \pi)^{3}}\left[\delta_{i l} \delta_{k m}+\delta_{i m} \delta_{k l}-\frac{2}{3} \delta_{i k} \delta_{l m}\right] \\
& \times \delta\left(\mathbf{k}_{1}+\mathbf{k}_{2}\right) \delta\left(t_{1}-t_{2}\right) .
\end{aligned}
$$

The conservation law (4) becomes

$$
\mathbf{k} \cdot \mathbf{v}_{\mathbf{k}}=0
$$

and the Navier-Stokes eq (5) becomes (on omission of the time-derivative)

$$
0=-i \mathbf{k} p_{\mathbf{k}}-\eta k^{2} \mathbf{v}_{\mathbf{k}}+i \mathbf{k} \cdot \mathbf{S}(\mathbf{k}) .
$$

The pressure is found by multiplying eq (11) by $\mathbf{k}$,

$$
p_{k}=\mathbf{k} \mathbf{k}: \mathbf{S}(\mathbf{k}) / \mathbf{k}^{2} .
$$

Then the velocity is

$$
\mathbf{v}_{\mathbf{k}}=\frac{1}{\eta k^{2}}\left\{i \mathbf{k} \cdot \mathbf{S}(\mathbf{k})-i \mathbf{k} \mathbf{k} \mathbf{k}: \mathbf{S}(\mathbf{k}) / k^{2}\right\} .
$$

The velocity correlation is found easily from eqs (13) and (9),

$$
\left\langle\mathbf{v}\left(\mathbf{k}_{1}, t_{1}\right) \mathbf{v}\left(\mathbf{k}_{2}, t_{2}\right)\right\rangle=\frac{4 k_{B} T}{\eta} \frac{1}{(2 \pi)^{3}} \frac{1}{k_{1}^{2}} \delta\left(\mathbf{k}_{1}+\mathbf{k}_{2}\right) \delta\left(t_{1}-t_{2}\right) .
$$

The mean force $\langle\mathbf{F}(t)\rangle$ vanishes, because the mean fluctuating stress tensor vanishes. But the correlation in the fluctuating force does not vanish. This is precisely the quantity we need for the present calculation.

Let us rewrite eq (7) using Fourier components,

$$
\mathbf{F}(t)=6 \pi \eta a \int d^{3} k \frac{\sin k a}{k a} \mathbf{v}(\mathbf{k}, t) .
$$

On using eq (14) we obtain

$$
\begin{aligned}
\langle\mathbf{F}(0) \cdot \mathbf{F}(t)\rangle & =(6 \pi \eta a)^{2} \int d^{3} k_{1} \int d^{3} k_{2} \\
\times & \frac{\sin k_{1} a}{k_{1} a} \frac{\sin k_{2} a}{k_{2} a}\left\langle\mathbf{v}\left(\mathbf{k}_{1}, 0\right) \cdot \mathbf{v}\left(\mathbf{k}_{2}, t\right)\right\rangle \\
& =18 \frac{k_{B} T \eta a^{2}}{\pi} \int d^{3} k \frac{1}{k^{2}}\left(\frac{\sin k a}{k a}\right)^{2} \delta(t) .
\end{aligned}
$$

The remaining integral is elementary, and the result is

$$
\langle\mathbf{F}(0) \cdot \mathbf{F}(t)\rangle=36 \pi k_{B} \operatorname{T\eta } a \delta(t) .
$$

To complete the derivation, we need only to put (17) into (3). Because the time integration runs from $t=0$ to $t=\infty$, and not from $t=-\infty$ to $t=\infty$, we pick up only half of the delta function. This gives

$$
\zeta=6 \pi \eta a,
$$

in complete agreement with Stokes' law. 
It should be noted that we have not actually derived Stokes' law from the time-correlation formula. In fact, Stokes' law (or rather, Faxen's generalization) played an essential role in the preceding discussion. Nevertheless, the argument is not circular; the generalized Navier-Stokes-Langevin equation, together with eq (6) for the correlation in the fluctuating stress tensor, cannot be obtained from purely hydrodynamic arguments. Similarly, the time-correlation formula for the friction constant cannot be obtained from hydrodynamics. A statistical theory is needed.

If one wished to derive Stokes' law directly from molecular theory, the most natural procedure would be first to derive the fundamental hydrodynamic laws from molecular theory. But having obtained the hydrodynamic laws, one might as well use them to get Stokes' law. In other words, the time-correlation formula is hardly necessary. All we have done in this article is to show how the time-correlation formula gives the same results as the hydrodynamic theory under conditions where the hydrodynamic theory is already available.

\section{References}

[1] J. G. Kirkwood, J. Chem. Phys. 14, 180 (1946).

[2] J. L. Lebowitz and E. Rubin, Phys. Rev. 131,2381 (1963). For a somewhat different derivation leading to the same result, see R. Zwanzig, J. Chem. Phys. 40, 2527 (1964).

[3] M. S. Green, J. Chem. Phys. 19, 1036 (1951).

[4] R. M. Mazo, J. Chem. Phys. 35, 831 (1961).

[5] L. D. Landau and E. M. Lifshitz, Fluid Mechanics (Pergamon Press, London, 1959). Chapter XVII deals with hydrodynamic fluctuations.

[6] M. S. Green, J. Chem. Phys. 20, 1281 (1952); 22, 398 (1954). A more rigorous treatment may be found in R. Zwanzig, Phys. Rev. 124, 983 (1961).

[7] H. Faxen, Ark. Mat. Astronom. Fys. 18, No. 29 (1924). Faxen's work, and more modern developments, are discussed by R. Berker in Volume VIII/2 of the Encyclopedia of Physics, edited by S. Flügge and C. Truesdell (Springer-Verlag, Berlin, 1963).

(Paper 68B4-126) 